\title{
Exploring positive adjustment in HIV positive African women living in the UK
}

\author{
Bridget Dibb* and Tamra Kamalesh \\ Department of Psychology, Brunel University, Uxbridge, UK \\ (Received 11 October 2010; final version received 14 June 2011)
}

\begin{abstract}
Research into living with HIV/AIDS has to date mainly focused on quality of life and there is little on the adjustment process for this group. The numbers of African women living with HIV/AIDS in the UK is growing and yet little is known about the adjustment experience for these women. This study explored aspects of positive adjustment to living with HIV/AIDS among a sample of African women living in London, UK. Transcripts of semi-structured interviews with 12 women were analysed using interpretative phenomenological analysis (IPA). Two superordinate themes emerged inductively from the data: positive changes in coping (subthemes: positive interpretation of their situation and positive behavioural changes) and positive growth since the HIV diagnosis (subthemes: changes in the value of life and, changes in goals and opportunities). While these women acknowledged the negative impact of living with HIV/AIDS, all participants mentioned changes in health behaviours to help regain mastery of their lives and comparing with others better-off and worse-off was used to enhance self-esteem and view their situation positively. The data show evidence for Taylor's Cognitive Adaptation Theory.
\end{abstract}

Keywords: HIV/AIDS; positive adjustment; positive growth; qualitative; IPA

\section{Introduction}

Women currently make up one third of the population living with HIV/AIDS in the UK (Health Protection Agency [HPA], 2008) with over $70 \%$ of HIV diagnosed women reported as black African origin (Department of Health, 2005). Although limited, research into the experiences of black Africans living with HIV/AIDS in the UK has largely revealed evidence of the difficult and negative aspects of living with HIV, as well as the additional difficulties of living life as an immigrant (Anderson \& Doyal, 2004; Dodds, 2006; Doyal \& Anderson, 2005b; Flowers et al., 2006; Ndirangu \& Evans, 2008). However, research in the US shows there can be positive aspects to coping with and adjusting to traumatic events, including an HIV diagnosis, and that continued personal distress and growth often co-exist (Dunbar, Mueller, Medina, \& Wolf, 1998; Gloerson et al., 1993; Littlewood, Vanable, Carey, \& Blair, 2008; Milam, 2006; Schaefer \& Coleman, 1992; Schwartzberg, 1993; Siegel \& Schrimshaw, 2000; Tedeschi \& Calhoun, 2004; Updegraff, Taylor, Kemeny, \& Wyatt, 2002).

One theory offering an explanation for the relationship between positive adaptation and growth is Taylor's (1983) Cognitive Adaptation Theory. This theory views people as adaptable, self-protective and functional in the face of adversity. The theory suggests that stressful events challenge core beliefs that people are then motivated to restore: a sense of meaning (achieved by positive growth), a sense of mastery (achieved by changes in controllable aspects of life, for example, diet and exercise), and self-esteem (achieved through downward social comparison) (Taylor, 1983).

There is some suggestion that positive adjustment may be present in people with HIV/AIDS. For example, Flowers et al. (2006) found some evidence of positive "wonderful" changes resulting from an HIV diagnosis in male and female African participants. Likewise, receiving care and medication that would be unavailable in their own country has been reported as a positive experience for African women living with HIV in the UK (Anderson \& Doyal, 2004; Ndirangu \& Evans, 2008). The benefits of practical coping on quality of life was reported in a study with HIV-positive African women (Onwumere, Holttum, \& Hirst, 2002). Ridge, Williams, Anderson and Elford (2008) found that faith and prayer promoted hope and a positive state of mind in black African HIV-positive men and women living in London. Support for positive growth has been found in a diverse range of cultures and traumatic experiences, including people with cancer (Ho, Chan, \& Ho, 2004), survivors of war (Powell, Rosner, Butollo, Tedeschi, \& Calhoun, 2003), and people living with HIV/AIDS (Anderson \& Doyal, 2004; Korner, 2007; Luszczynska, Sarker \& Knoll, 2006). A study looking at the experience of living with HIV in African

*Corresponding author. Email: bridget.dibb@brunel.ac.uk 
women have found a greater sense of spirituality since the onset of their HIV (Doyal \& Anderson, 2005b).

Because of the preponderance of women from sub-Saharan Africa living with HIV/AIDS in the UK (Department of Health, 2005) it is important to understand the experiences of these women. However, a recent literature search (Web of knowledge July, 2010) has found a lack of in-depth studies investigating positive adjustment and growth within this group. The purpose of the present study was therefore to explore the experience of living with HIV/AIDS for African women living in the UK with a view to understanding positive adjustment and growth within this group, knowledge which will be beneficial to health professionals promoting self-help programmes for people with HIV. The question this study sought to answer was: do HIV positive African women living in the UK positively adjust to living with HIV and, if so, in what way this adjustment may occur?

\section{Method}

As the aims of this study were explorative a qualitative design was employed using interpretative phenomenological analysis (IPA) to analyse the data and so understand the individual's experience.

\section{Sampling and recruitment}

Participants were recruited using opportunistic and snowball sampling (via referral) through a charity offering support to people with an HIV-positive diagnosis. Criteria for inclusion, apart from being diagnosed HIV-positive for $>6$ months, were being born and raised to African parents in Africa until at least age 12 and a fluency in spoken English.

\section{Participants}

Using the IPA framework the participants consisted of a homogenous sample of 12 women (six Ugandans and six Zimbabweans). Participants' ages ranged from 32 to 60 years (mean: 46.5 years). All had contracted HIV through heterosexual intercourse and had been diagnosed 3-16 years ago (mean: 7 years). All were on HIV medication. Participants had lived in the UK between 4 and 15 years (mean: 7.5 years). Eleven participants had children; 11 had completed high school and five had a college education. Seven participant's applications for residency were being reviewed by the Home Office, two were awaiting residency papers, and three had residency.

\section{Procedure}

After receiving ethical approval, semi-structured interviews (conducted by TK) were used to explore each woman's personal experience of living with HIV. The interview schedule followed an exploratory path asking the participants what it was like to live with HIV/AIDS, with a focus on whether they felt they had changed as a result of HIV, and in what way this change had occurred (see Table 1 for a list of the questions asked). As smaller sample sizes are needed with a qualitive study than with quantitative studies, in order to achieve data saturation twelve participants were recruited. The interviews took place in the participants' homes and lasted between 45 and 90 minutes. Participants received a $£ 10$ voucher for their time.

\section{Data analysis}

Interpretative Phenomenological Analysis (IPA; Smith, Jarman, \& Osborn, 1999) provides an in-depth understanding of the participants' experience and does not aim to generalise the findings to all individuals. It has been used successfully in sexual health studies (Flowers et al., 2006) and was viewed as the most appropriate method of analysis for this study. IPA allows the inductive development of themes within and across interviews. After an in-depth reading and rereading of the transcripts, initial coding (conducted by TK) identified groups of meaning within the text. Categories of meaning were

Table 1. Interview questions.

Can you tell me about what it's like living with HIV?

Do you feel that having HIV has had an impact on your life?

- In what way?

- What was it like when you first found out?

Do you think having HIV has changed you as a person in anyway? How?

Do you do anything differently now?

What would you say are the most important things in your life at the moment? How is this different to how you felt before?

Some people also say there have been positive aspects about their diagnosis, would you agree with that? (If yes) Can you tell me what some of these positive experiences have been? 
then grouped to form subthemes and super-ordinate themes (the thematic framework and categories was discussed by both authors). The approach acknowledges the researchers' influence and interpretation of the data.

\section{Results}

Inductive themes of adjustment and perceived growth were identified. While a number of changes were negative, the women also spoke of positive growth that had occurred since their HIV diagnosis which had enabled them to move forward with their lives optimistically. As the focus of this article is on positive adjustment, only the positive themes are presented here. Two super-ordinate themes were identified - positive changes in coping and positive growth since the HIV diagnosis (see Table 2).

\section{Super-ordinate theme - positive changes in coping}

Subtheme: positive interpretation of their situation

This theme reflects the positive coping strategies these women engaged in. Comparing themselves with others allowed the participants to hold a more positive future outlook on life, increased their confidence and increased their self-esteem. Both upward (where we compare with others who are better-off than ourselves) and downward comparison (where we compare with others who are worse-off than ourselves) were important here.

Elizabeth engaged in upward social comparison where she compared herself with someone coping well with HIV, enabling her to be aware of the shortcomings of her own behaviour and inspired her to cope better:

She told me, [...] 'I'm positive as well' but she was looking good. I said 'Oh my god' Then I knew my life because, if a person like her, looking good as she is, then I should do something and stop moaning about myself, so I stopped. (Elizabeth)

Another participant, Jessica, used downward social comparison comparing herself with people not infected with HIV but still interpreting this positively. This was a potentially threatening comparison as she was HIV-positive, while the targets (the people she compared herself with) were not, yet she re-interpreted the situation so that she was the one who was better off. This strategy removed the stress associated with worry and allowed her to feel positive about her situation:

You only get infected with HIV once, so you don't have to worry about it anymore anyway. So for those people who are negative, they have to worry not to get it. Me, I don't worry about it anymore. All I have to worry is to look after myself. Not get any, like, re-infected, by using unprotected sex. Just look after myself really. Yes, that's the positive part, one of them. (Jessica)

Jane also used downward social comparison, compared herself with those less fortunate with regards to their illness severity, interpreted the comparison positively and felt better about her own situation:

Sometimes you see people [at the support groups] who are very ill, more than you, and you think 'Oh, I'm much better off'. (Jane)

\section{Subtheme: positive behavioural changes}

Although many of the women found taking medication every day an unwanted reminder of their illness, many had adopted other healthier behaviours since their diagnosis. Sticking to a strict medical regime and seeing the medication working encouraged a sense of coping and control over their illness.

I feel I really cope. I feel I am positive to live with HIV because if I'm not positive I think everything won't work. And why I am very much positive, the treatment is working. The medication I am taking is working. (Jane)

Jennifer took control by engaging in health behaviours. She took precautions with the food she ate and the water she drank, and did what she could do to protect herself from malaria, revealing her beliefs about her immune system.

When I travel [...] to Zimbabwe, before I knew about my diagnosis I would just drink water when I go to the village but now because I know that my immunity is suppressed I buy those creams to protect me from

Table 2. Table of themes.

\begin{tabular}{ll}
\hline Superordinate theme & \multicolumn{1}{c}{ Subtheme } \\
\hline Positive changes in coping & $\begin{array}{l}\text { Positive interpretation of their situation } \\
\text { Positive behavioural changes } \\
\text { Changes in the value of life } \\
\text { Cositive growth since the HIV diagnosis }\end{array}$ \\
& Changes in goals and opportunities \\
\hline
\end{tabular}


getting malaria ... I also make sure the water I drink, I boil it first which I never used to do... And I don't just pick food and eat, just picking, I take precautions as well because I don't want to affect that immune system that is already suffering. (Jennifer)

Practising safe sex was another behavioural change mentioned by participants. Jessica was particularly forthright about this:

For someone if they accept me that I'm HIV positive they will be prepared to use condoms and if they're not prepared they go away. (Jessica)

For Faith, not being married has given her more control over using condoms with her partner:

If you are married you don't have control over what your partner is doing so I actually feel like I'm clean because I'm in control of these condoms. (Faith)

Violet, too, had become much more careful about practicing safe sex since her HIV diagnosis:

But when I came into this country seeing the symptoms [...] I started to think maybe my husband died of HIV, so when I met this guy I was so careful, we used condoms. (Violet)

\section{Super-ordinate theme-positive growth since the HIV diagnosis}

This super-ordinate theme shows how the participants experienced positive growth in their lives since the onset of their HIV.

\section{Subtheme: changes in the value of life}

Participants had clearly found more meaning and value in life because of their HIV diagnosis. The burden of other life factors, above and beyond HIV, is likely to have also influenced this discovery.

Emma and Elizabeth talked of how their value of life had changed since being diagnosed with HIV; Emma now perceived her time to be limited:

Before I was just living. There was no meaning to life ... But now I know my time is limited. I need to do things. I need to have an impact on some things. [...] before I wasn't doing what I wanted, I was living for others. Right now I am living for me, me, me, me. Myself ... I found who I am. I found out who I am. (Emma)

Now I can think more about my life, because of HIV. Back then, when I was not, I was not thinking about it. I could live, just like that, but now I am more careful ... I think about my life, I take my life as very important ... Living with HIV ... I managed to value my life. (Elizabeth)

\section{Subtheme: changes in goals and opportunities}

The participants reported that HIV had given them a new set of goals and newfound opportunities, largely related to helping those newly diagnosed with HIV, or within the educational or care sector. Below Violet explained she now felt great empathy with children diagnosed with HIV, which she attributed to her own experience with HIV:

I've got ambition to work in various areas, although I am targeting young children and HIV people, I've got experience with young children because before I had my own nursery. The most part of it that pains me is to see a young child being diagnosed with HIV so I need to be counsellor towards that. (Violet)

After being diagnosed as HIV-positive, Jane could no longer work as a midwife but found a different way of using her skills:

There are many different things you can do. You can divert to looking after special people with HIV. I think there's many courses which I can take, definitely take, when they give me status. [...] I have to [...] look after maybe people with the same condition. Even if it's in a hospital but on that ward I can work there. (Jane)

Her comment "even if it's in a hospital ..." indicates the limitations she has experienced within her job since her HIV diagnosis, but she has found that she can work with patients living with HIV/AIDS.

Although Emma said that prior to diagnosis she used to fear people living with HIV, she discovered a personal strength which she utilised in her voluntary work in the HIV community. She also acknowledged the satisfaction she experienced from helping others "I feel nice inside me because I've been there":

Because of what I went through I feel so strengthened to support people. I feel nice when I am supporting somebody who has just been diagnosed, who is really confused and, you know, I feel good to talk to her and give her, you know, strengthen her, you know. I feel nice inside me because I've been there. I have experienced what she's talking about and I give them assurance 'things will be okay, don't worry'. (Emma)

\section{Discussion}

These results provide evidence of positive coping and growth experienced by African women living with HIV in London. While all women had experienced negative consequences of HIV, such as stigma and psychological distress, all had also found that their diagnosis had contributed something positive to their lives as well. 
The themes emerged inductively from the transcripts. Themes indicate resilience where the participants mentioned methods used to re-build self-esteem, life meaning, and control over their illness, offering support for Taylor's (1983) Cognitive Adaptation Theory (CAT).

The participants coped positively with their illness by positively interpreting their situation and making behaviour changes.

Positively interpreting their situation was achieved with both downward comparison, as proposed by the CAT (Taylor, 1983) to increase self-esteem, and upward comparison, proposed later by Taylor and Lobel (1989), to give hope for things to improve, were reported. The positive effects of social comparison have been found in other illness groups, for example, cancer (Van der Zee, Buunk, Sanderman, Botke, \& Van den Bergh, 2000), and Ménière's disease (Dibb \& Yardley, 2006). This positive interpretation of the comparison information supports adjustment theorists who suggest we have a natural tendency to interpret the world positively and adapt to adverse circumstances (Schwartz \& Sprangers, 2000).

The "Positive changes in coping" subtheme, "positive behavioural changes", shows positive coping and a positive attitude to managing health decisions, again supporting the CAT (Taylor, 1983), where the onset of a crisis leads to a need to regain control (mastery). Siegel and Schrimshaw (2000) found a similar theme in their research with people with HIV, which they labelled "motivation to make health behaviour changes"; in the current study, however, the participants took this theme further, beyond motivation, to initiate behaviour change. This theme also supports Onwumere et al. (2002) who found that practical changes were associated with positive outcomes in a sample of African women with HIV. It was also evident that these behavioural changes were improving their perceptions of control over their lives.

The participants also perceived change since their diagnosis, as evidenced by the superordinate theme "positive growth since the HIV diagnosis", again supporting the CAT (Taylor, 1983) where perceiving positive growth is proposed to give meaning to the situation. Meaning was gained with positive growth in regard to the value they attached to life and their goals and perceived opportunities. This is supported by another study with women with HIV living in the US where changes the meaning of life was also found (Siegel \& Schrimshaw, 2000). Consistent with previous research, all of the women had also managed to reformulate their life goals to incorporate HIV allowing them to see different opportunities available to them and give new meaning to their lives (Doyal \&
Anderson, 2005a; Siegel \& Schrimshaw, 2000). Involvement within the HIV community allowed for the maintenance of these values, whilst also providing the opportunity to strengthen oneself as well as others (Dodds, Hickson, Chinouya, Chwaula, \& Westherburn, 2008). The importance of remaining part of a community could be considered even more important for people from African cultures than Western ones, with values of mutual care, belonging and interdependence considered highly important in these cultural groups (Singelis, 1998; Triandis, 1995).

This study was conducted with a small sample of women to gain insight into their subjective experiences of living with HIV/AIDS; thus, there are limitations to the generalisability and applicability of these findings to the wider population of African women in London and elsewhere. The women were recruited using opportunistic and snowball sampling, through a support group network, and could be significantly different from women not using support groups.

This study has provided insight into the ability of HIV positive African women living in London to create strategies to cope and live positively with HIV/ AIDS. The findings provide strong support for the CAT (Taylor, 1983), with regaining mastery, enhancing self-esteem, and finding meaning evident in the data.

Strategies used by this sample that resulted in regaining control, self-enhancing, and giving meaning may be important to health professionals developing self-management programmes for people with HIV. Further work looking at the value placed on specific forms of recovery and growth by African people living with HIV/AIDS would add value to this underresearched topic and allow a better understanding of adjustment cross-culturally.

\section{References}

Anderson, J., \& Doyal, L. (2004). Women from Africa living with HIV in London: A descriptive study. AIDS Care, 16(1), 95-105.

Department of Health. (2005). HIV and AIDS in African communities: A framework for better prevention and care. London: Department of Health.

Dibb, B., \& Yardley, L. (2006). How does social comparison within a self-help group influence adjustment to chronic illness? A longitudinal study. Social Science and Medicine, 63(6), 1602-1613.

Dodds, C. (2006). HIV-related stigma in England: Experiences of gay men and heterosexual African migrants living with HIV. Journal of Community and Applied Social Psychology, 16, 472-480.

Dodds, C., Hickson, F., Chinouya, M., Chwaula, J., \& Westherburn, P. (2008). The knowledge, the will and the 
power: A plan of action to meet the HIV prevention needs of Africans living in England. UK: Sigma Research.

Doyal, L., \& Anderson, J. (2005a). My heart is loaded: African women with HIV surviving in London Report of a Qualitative Study. Retrieved from http:// www.tht.org.uk/informationresources/publications/policy reports/myheart584.pdf (accessed 17 July 2008).

Doyal, L., \& Anderson, J. (2005b). My fear is to fall in love again ...' How HIV-positive African women survive in London. Social Science and Medicine, 60, 1729-1738.

Dunbar, H.T., Mueller, C.W., Medina, C., \& Wolf, T. (1998). Psychological and spiritual growth in women living with HIV. Social Work, 43(2), 144-154.

Flowers, P., Davis, M., Hart, G., Rosengarten, M., Frankis, J., \& Imrie, J. (2006). Diagnosis and stigma and identity amongst HIV positive Black Africans living in the UK. Psychology and Health, 21(1), 109122.

Gloerson, B., Kendall, J., Gray, P., McConnell, S., Turner, J., \& Lewkowicz, J.W. (1993). The phenomena of doing well in people with AIDS. Western Journal of Nursing Research, 15(1), 44-58.

Health Protection Agency (HPA). (2008). Communicable Disease Surveillance Centre (HIV and STI Department) and the Scottish Centre for Infection and Environmental Health: 'Unpublished Surveillance Tables No. 77, 07/4', March 2008. Retrieved from http://www.hpa.org.uk/infections/topics_az /hiv_and_ sti/Stats/default.htm (accessed 1 April 2008).

Ho, S.M.Y., Chan, C.L.W., \& Ho, R.T.H. (2004). Posttraumatic growth in Chinese cancer survivors. PsychoOncology, 13(6), 377-389.

Korner, H. (2007). Negotiating cultures: Disclosure of HIV-positive status among people from minority ethnic communities in Sydney. Culture Health \& Sexuality, 9(2), 137-152.

Littlewood, R.A., Vanable, P.A., Carey, M.P., \& Blair, D.C. (2008). The association of benefit finding to psychosocial health behaviour adaptation among $\mathrm{HIV}+$ men and women. Journal of Behavioural Medicine, 31(2), 145-155.

Luszczynska, A., Sarker, Y., \& Knoll, N. (2006). Received social support, self-efficacy, and finding benefits in disease as predictors of physical functioning and adherence to antiretroviral therapy. Patient Education and Counseling, 66, 37-42.

Milam, J. (2006). Posttraumatic growth and HIV disease progression. Journal of Consulting and Clinical Psychology, 74(5), 817-827.

Ndirangu, E., \& Evans, C. (2008). Experiences of African immigrant women living with HIV in the UK: Implications for health professionals. Journal of Immigrant Minority Health, http://www.springerlink. com/content/n23460w660761445 (Accessed 6 March 2008).

Onwumere, J., Holttum, S., \& Hirst, F. (2002). Determinants of quality of life in black African women with
HIV living in London. Psychology Health and Medicine, 7(1), 61-74.

Powell, S., Rosner, R., Butollo, W., Tedeschi, R.G., \& Calhoun, L.G. (2003). Post-traumatic growth after war: A study with former refugees and displaced people in Sarajevo. Journal of Clinical Psychology, $59,71-83$.

Ridge, D., Williams, I., Anderson, J., \& Elford, J. (2008). Like a prayer: The role of spirituality and religion for people living with HIV in the UK. Sociology of Health and Illness, 30(3), 413-428.

Schaefer, S., \& Coleman, E. (1992). Shifts in meaning, purpose, and values following a diagnosis of human immunodeficiency virus (HIV) infection among gay men. Special issue: Sexual transmission of HIV infection: Risk reduction, trauma, and adaptation. Journal of Psychology \& Human Sexuality, 5(1-2), 13-29.

Schwartz, C.E., \& Sprangers, M.A.G. (2000). Adaptation to changing health: Response shift in quality of life research. Washington, DC: American Psychological Association.

Schwartzberg, S.S. (1993). Struggling for meaning: How HIV-positive gay men make sense of AIDS. Professional Psychological Research and Practice, 24(4), 483-490.

Siegel, K., \& Schrimshaw, E.W. (2000). Perceiving benefits in adversity: Stress-related growth in women living with HIV/AIDS. Social Science and Medicine, 51, 1543-1554.

Singelis, T.M., (Ed.). (1998). Teaching about culture, ethnicity, and diversity: Exercises and planned activities. Thousand Oaks, CA: Sage.

Smith, J.A., Jarman, M., \& Osbourn, M. (1999). Doing interpretative phenomenological analysis. In M. Murray \& K. Chamberlain (Eds.), Qualitative health psychology: Theories and methods (pp. 218-240). London: Sage.

Taylor, S.E. (1983). Adjustment to threatening events: A theory of cognitive adaptation. American Psychologist, $38,1161-1173$.

Taylor, S.E., \& Lobel, M. (1989). Social comparison activity under threat: Downward evaluation and upward contacts. Psychological Review, 96, 569-575.

Tedeschi, R.G., \& Calhoun, L.G. (2004). Posttraumatic growth: Conceptual foundations and empirical evidence. Psychological Inquiry, 15(1), 1-18.

Triandis, H. (1995). Individualism and collectivism. Boulder, CA: Westview Press.

Updegraff, J.A., Taylor, S.E., Kemeny, M.E., \& Wyatt, G.E. (2002). Positive and negative effects of HIV infection in women with low socioeconomic resources. Personality and Social Psychological Bulletin, 28, 382394.

Van der Zee, K., Buunk, B.P., Sanderman, R., Botke, G., \& Van den Bergh, F. (2000). Social comparison and coping with cancer treatment. Personality and Individual Differences, 28, 17-34. 